\title{
Programming Biomimetically Confined Aptamers with DNA Frameworks
}

Xiuhai Mao, ${ }^{1, \#}$ Mengmeng Liu,,${ }^{2, \#}$ Lei Yan, ${ }^{1}$ Mengying Deng, ${ }^{3,4}$ Fan Li, ${ }^{1}$ Min Li, ${ }^{1}$ Fei Wang, ${ }^{5,6}$ Jiang Li, ${ }^{3,4}$ Lihua Wang, ${ }^{3,4}$ Yang Tian, ${ }^{2}$ Chunhai Fan, ${ }^{1,6}$ and Xiaolei Zuo ${ }^{1,6,}{ }^{*}$

${ }^{1}$ Institute of Molecular Medicine, Shanghai Key Laboratory for Nucleic Acid Chemistry and Nanomedicine, Renji Hospital, School of Medicine, Shanghai Jiao Tong University, Shanghai 200127, China

${ }^{2}$ Shanghai Key Laboratory of Green Chemistry and Chemical Processes, Department of Chemistry, School of Chemistry and Molecular Engineering, East China Normal University, Dongchuan Road 500, Shanghai 200241, China

${ }^{3}$ Division of Physical Biology, CAS Key Laboratory of Interfacial Physics and Technology, Shanghai Institute of Applied Physics, Chinese Academy of Sciences, Shanghai 201800, China

${ }^{4}$ Bioimaging Center, Shanghai Synchrotron Radiation Facility, Zhangjiang Laboratory, Shanghai Advanced Research Institute, Chinese Academy of Sciences, Shanghai 201210, China

${ }^{5}$ Joint Research Center for Precision Medicine, Shanghai Jiao Tong University Affiliated Sixth People's Hospital South Campus, Southern Medical University Affiliated Fengxian Hospital, Shanghai 201499, China

${ }^{6}$ School of Chemistry and Chemical Engineering, Frontiers Science Center for Transformative Molecules, Institute of Translational Medicine, Shanghai Jiao Tong University, Shanghai 200240, China 


\section{Experiments and Methods}

\subsection{Tetrahedral Framework nucleic acids formation :}

Four stoichiometric DNA strands, S1-S4 shown in the Table S1, were mixed in assembly buffer (10 mM Tris, $50 \mathrm{mM} \mathrm{KCl}$, and $15 \mathrm{mM} \mathrm{MgCl}_{2}, \mathrm{pH} \mathrm{8.0)}$. Then, this mixture was heated at $95^{\circ} \mathrm{C}$ for $5 \mathrm{~min}$ and then cooled at $4{ }^{\circ} \mathrm{C}$ for $30 \mathrm{~s}$ with a PCR machine (Applied Biosystems, US).

\subsection{Atomic force microscopy (AFM) imaging:}

Samples were first deposited on mica surfaces for 5 mins. AFM images were then taken in tapping mode in fluid mode on a Cypher ES (Asylum Research) using BL-AC40TS tips (Olympus). Typical scanning parameters were: scan rate $=1-2 \mathrm{~Hz}$, lines $=512$, amplitude set point $=150-300 \mathrm{mV}$, drive amplitude $=180-300 \mathrm{mV}$, integral gain $=18$.

\subsection{Gel Electrophoresis}

All DNA samples, including assembled Tetrahedral FNA, were analyzed by $10 \%$ Native PAGE (29:1 Acrylamide: bis-acrylamide mixture) using 1× TBE buffer and stained with Sybr Gold (Invitrogen, US).

\subsection{Zeta Potential measurement}

The zeta-potential of the FNA samples were measured with Zetasizer NANO-ZS (from Malvern Instruments). Five measurements were performed to obtain the average $\zeta$ potential of the samples.

\subsection{Capillary Electrophoresis}

All DNA sample were diluted to $50 \mathrm{nM}$ with $\mathrm{H}_{2} \mathrm{O}$ and analyzed in Qsep100 with high resolution cartridge kit $(\mathrm{S} 1)$.

\subsection{Purification of FNA and aptamer-FNA complex by SEC}

A SEC column (Phenomenex BioSec-SEC-S2000, 300 x $7.8 \mathrm{~mm}$ ) was used and all chromatograms were recorded at $260 \mathrm{~nm}$. A typical SEC mobile phase was $30 \mathrm{mM}$ Tris-HCl, pH 7.4, $450 \mathrm{mM} \mathrm{NaCl}$. Prepared DNA framework (50-500 $\mu \mathrm{L})$ were loaded and purified at a flow rate of $0.6 \mathrm{~mL} / \mathrm{min}$ with isocratic elution.

\subsection{Switching Equilibrium constant (Ks) determination for aptamer}

The DNA were modified with FAM and Dabcyl at 5' end and intermedia of DNA stand, respectively, shown in the Table S1. This experiment was obtained using a FL1000 (Edinberg Technologies) with an excitation wavelength at $494( \pm 5) \mathrm{nm}$ and an acquisition wavelength at $520( \pm 5) \mathrm{nm}$.

The fluorescence $v s$ anti-aptamer stand plot were normalized into Ks (fraction folded) using the following equation:

$$
K s=(F f-F b) / F b
$$


Where $F f$ and $F b$ correspond to the fluorescence values of the duplex stands (nonnative state) and native aptamer obtained from their baselines, respectively.

\subsection{Thermo-melting determination}

The DNA were modified with FAM and BHQ-1 at 5' end and intermedia of DNA stand, respectively. Thermo-melting curves were obtained using a FL1000 (Edinberg Technologies) with an excitation wavelength at $532( \pm 5) \mathrm{nm}$ and an acquisition wavelength at $561( \pm 5) \mathrm{nm}$. The temperature raised to $85{ }^{\circ} \mathrm{C}$ from $25{ }^{\circ} \mathrm{C}$ at a rate of $1.0^{\circ} \mathrm{C} \cdot \mathrm{min}^{-1}$ using a total reaction volume of $500 \mu \mathrm{l}$ in a quartz cuvette. To limit the evaporation of the sample during the experiment, the top cover of quartz cuvette was further wrapped with thick parafilm. All the aptamer-FNA samples were firstly purified with HPLC and cooled to room temperature in the quartz cuvette for 10 mins before heating to $95^{\circ} \mathrm{C}$.

For the thermodynamic analysis of the melting profiles a two-state model and a temperature independent $\Delta H^{\circ}(\triangle C p=0)$ were assumed. The fluorescence $v s$ temperature plots were normalized into Ks (equilibrium constant) vs temperature exploiting the previously calculated baselines and using the following equation:

$$
K s=(F f-F t) /(F t-F b)
$$

Where Ft corresponds to the fluorescence signal at a given temperature, $F f$ and $F b$ correspond to the fluorescence values of the unfolded state and folded state obtained from their baselines for aptamer, respectively.

All the reported melting curves have been normalized through the use of the derivation of van't Hoff model by plotting the natural logarithm of the equilibrium constant $K s$ as a function of the reciprocal of the temperature $\left(1 / \mathrm{T}\right.$ in $\left.\mathrm{K}^{-1}\right)$. For the analysis the interval of $\alpha$ between 0.10 and 0.90 where $K s$ values are more precise was selected. To estimate the enthalpic and entropic contribution for the aptamer formation, the data were fitted with the following equation:

$$
\ln (K s)=-\Delta H / R^{*}(1 / T)+\Delta S / R
$$

\section{9 smFRET characterization of folding and unfolding process for the aptamer}

Single-molecule FRET experiments were performed on a Nikon N-STORM system. All smFRET movies were acquired at $50 \mathrm{~ms}$ time resolution using an intensified CCD camera. For each sample, tetrahedral FNA labelled with biotin was modified onto the $29 \mathrm{mM}$ glass bottom dishes (Cellvis, Catalog \#D29-20-1-N), which were pre-coated with biotinylated-bovine serum albumin followed by streptavidin. The diluted samples $(1 \mathrm{nM}, 10 \mu \mathrm{l})$ were dropped in the dish and incubated for $10 \mathrm{~min}$, followed by rinsing with assemble buffer three times. Doubly labeled aptamer on the tetrahedral FNA were excited with $561 \mathrm{~nm}$ green laser for measuring FRET and later with $640 \mathrm{~nm}$ red laser to check for the presence of Cy5 (acceptor) in order to distinguish low/zero FRET molecules from the ones missing an acceptor fluorophore. To extend the lifetime of fluorophores and to prevent photoblinking of the dyes, an enzymatic oxygen scavenging system, which consists of $5 \mathrm{mM}$ protocatechuic acid (PCA) and $50 \mathrm{nM}$ protocatechuate-3,4-dioxygenase (PCD) along with $2 \mathrm{mM}$ Trolox, was added into the 
buffer. Then the videos were analyzed using ImageJ and Python to quantify the time when the CY5 molecules excited by Cy3 molecule.

The raw movies were processed using ImageJ to extract smFRET time traces and later analyzed using custom-written Python. Individual smFRET time traces displaying single-step photobleaching, a signal-to-noise ratio of $>4: 1$, a total (donor-acceptor) fluorescence intensity of $>300$ (arbitrary units), and a total fluorescence duration of $>10$ s was manually selected.

Cumulative histograms of single-molecule survival times were fit to an exponential-decay model to determine parameters for the individual decay lifetimes with the following equation:

$$
\mathrm{N}=\mathrm{Ae}^{-\mathrm{t} / \tau}
$$

Fitting was performed using nonlinear least-squares with squared residuals weighted by the statistical error, represented by $1 / \mathrm{N}(\mathrm{t})$, where $\mathrm{N}(\mathrm{t})$ is the histogram-bin count at time bin $\tau$. Although the magnitudes of the residuals from a two-exponential fit exceed the expected statistical uncertainty in the histogram-bin counts, a model with three exponential components showed evidence of overfitting of the data.

\subsection{Length prediction for the aptamer in fold and unfold state}

In order to predict the length of aptamer in native and nonnative conformation, a selfavoiding random walk path constructed by a unit building block is demonstrated via Monte Carlo simulation. In details, each DNA base is presented as a hard sphere monomer, which is linked to each other with fixed bond length (1.7 base for single strand and 1 base for double strand).

For the nonnative aptamer with random coli structure, the position is derived from two random numbers defining polar angle coordinates in relation to the previous monomers. As the total structure is built monomer by monomer, it is crucial to keep track of the exact positioning of each element. Generally, after one initial monomer is deployed, all the following ones are added sequentially to link to the previous monomer, but with a random angle. The position of each spheres, as well as the formed bond between two DNA bases, must not overlap. In case that the chosen angles result in an overlap with one of the previous monomers, the addition is dismissed as well. In addition, a "fail counter" is increased. If that counter exceeds a defined threshold value, for instance because the chain end is trapped in a cavity, the monomer units of the current fragment are removed and the fragment is started again. Once we produced a serial of ordinate for these DNA base, we determine the length following the equation:

$$
\begin{array}{r}
\mathrm{L}=\max \left[\operatorname{sqrt}\left(\left(\mathrm{x}_{\mathrm{i}}-\mathrm{x}_{1}\right)^{2}+\left(\mathrm{y}_{\mathrm{i}}-\mathrm{y}_{1}\right)^{2}\right)\right] \\
\mathrm{i}=1,2, \sim, \mathrm{N} ;
\end{array}
$$

For the native aptamer with clover structure, the native conformation was separated into three segments: double stand and two hairpin structures. Firstly, we generated the coordinates for the inner six-membered ring with double stand DNA as initial monomers. Of note, the fixed distance between the adjacent points are 1.7 base pair for different segments and 1.0 base pair for the stem, respectively. Then, all the following monomers of DNA are added sequentially to link to the previous monomer 
in the six-membered ring of the stem. For the remaining hairpin domain, a stem structure with double strand was firstly generated, then a loop structure with five points were generated following the last pair of the stem. Once we produced a serial of ordinate for these DNA base, we determine the length following the equation 5 (Note: the predicted length results for native and nonnative aptamer are appended in the supplementary data 1and 2 , respectively).

\subsection{Entropy prediction for FNA confinement}

A simplistic decomposition emphasizes the fact that the entropy contribution for the folding process of aptamer is determined by the difference in the weight of the most probable configuration between the two conformation:

$$
\Delta S=k T \ln \left(\sum W_{\text {native }} / \sum W_{\text {nonnative }}\right)
$$

where $\mathrm{W}$ is the weight of the most probable configuration. Compared with outside configuration, the conformational entropy of aptamer suffers more restriction from the FNA scaffold, and the confinement-induced change in the $\mathrm{W}^{\mathrm{FNA}}$, becomes

$$
\begin{aligned}
W_{\text {native }} \text { inside }^{\text {inside }} & =\alpha_{\text {native }} * W_{\text {native }}^{\text {outside }} \\
\text { and } W_{\text {nonnative }} & =\alpha_{\text {nonnative }} * W_{\text {nonnative }}^{\text {outside }}
\end{aligned}
$$

where $\alpha$ is the conformation restriction from FNA confinement. Thus, following Eqs. s6 and s7, the confinement-induced change in the energy entropy between fold and unfold states, $d S_{F N A}$, becomes

$$
\begin{aligned}
\Delta S^{F N A} & =\Delta S_{\text {inside }}-\Delta S_{\text {outside }} \\
& =k T^{*}\left(\ln \left(\sum W_{\text {native }} \text { inside } / \sum W_{\text {nonnative }}^{\text {inside }}\right)-\ln \left(\sum W_{\text {native }} \text { outside } \sum W_{\text {nonnative }^{\text {outside }}}\right)\right) \\
& =k T^{*} \ln \left(\sum \alpha_{\text {native }} / \sum \alpha_{\text {nonnative }}\right)
\end{aligned}
$$

For the prediction, the coordinates for the FNA and the start point for the aptamer were firstly generated following the design. Generally, for a DNA with a fixed starting point and length, its rotational angel directly determined its position relative to FNA. Therefore, through randomly picking polar angle coordinates via Monte-Carlo method, we determined the proportion of aptamer staying in the FNA based on the predicted length in the supplementary Method 1.10. 


\section{Materials}

Table S1. DNA sequences

\begin{tabular}{|c|c|}
\hline Oligo & Sequence (5'----3') \\
\hline $\mathrm{S} 1$ & $\begin{array}{l}\text { C(8th)CAGGC(13th)AGTTGAG(20th)ACGAACATTCCTAA } \\
\text { GTCTGAAATTTATCACCCGCCATAGTAGAC(1st)GTATC } \\
\text { A }\end{array}$ \\
\hline S2 & $\begin{array}{l}\text { CTTGCTACACGATTCAGACTTAGGAATGTTCGACATG } \\
\text { CGAGGGTCCAATACCGACGATTACAG }\end{array}$ \\
\hline $\mathrm{S} 3$ & $\begin{array}{l}\text { GGTGATAAAACGTGTAGCAAGCTGTAATCGACGGGAA } \\
\text { GAGCATGCCCATCCACTACTATGGCG }\end{array}$ \\
\hline S4 & $\begin{array}{l}\text { CCTCGCATGACTCAACTGCCTGGTGATACGAGGATGG } \\
\text { GCATGCTCTTCCCGACGGTATTGGAC }\end{array}$ \\
\hline $\mathrm{CP}$ & ССTTCAATGAAGTGGGTCGAAAGGAGACAAGG \\
\hline Anti-CP & CCTTGTCTCCTTTCGACCCACTTCATTGAAGG \\
\hline $\begin{array}{l}\text { Biotin-TEG-S2 } \\
\text { for smFRET }\end{array}$ & $\begin{array}{l}\text { Biotin-TEG- } \\
\text { CTTGCTACACGATTCAGACTTAGGAATGTTCGACATG } \\
\text { CGAGGGTCCAATACCGACGATTACAG }\end{array}$ \\
\hline $\begin{array}{l}\text { Biotin-TEG S3 } \\
\text { for smFRET }\end{array}$ & $\begin{array}{l}\text { Biotin-TEG- } \\
\text { GGTGATAAAACGTGTAGCAAGCTGTAATCGACGGGAA } \\
\text { GAGCATGCCCATCCACTACTATGGCG }\end{array}$ \\
\hline $\begin{array}{l}\text { Biotin-TEG S4 } \\
\text { for smFRET }\end{array}$ & $\begin{array}{l}\text { Biotin-TEG- } \\
\text { CCTCGCATGACTCAACTGCCTGGTGATACGAGGATGG } \\
\text { GCATGCTCTTCCCGACGGTATTGGAC }\end{array}$ \\
\hline $\begin{array}{l}\text { CP-S1-in for } \\
\text { measuring } K s\end{array}$ & $\begin{array}{l}\text { (FAM)CCTTCAATGAAGTGGGTCGAAAGGAGACAAG } \\
\text { G(Dabcyl)CCAGGCAGTTGAGACGAACATTCCTAAGTCT } \\
\text { GAAATTTATCACCCGCCATAGTAGACGTATCA }\end{array}$ \\
\hline $\begin{array}{l}\text { CP-S1-out for } \\
\text { measuring } K s\end{array}$ & $\begin{array}{l}\text { (FAM)CCTTCAATGAAGTGGGTCGAAAGGAGACAAG } \\
\text { G(Dabcyl)CAGTTGAGACGAACATTCCTAAGTCTGAAAT } \\
\text { TTATCACCCGCCATAGTAGACGTATCACCAGG }\end{array}$ \\
\hline CP1-S1-in & $\begin{array}{l}\text { (FAM)GCCTTCAATGAAGTGGGTCGAAAGGAGACAA } \\
\text { GGC(Dabcyl)CCAGGCAGTTGAGACGAACATTCCTAAGT } \\
\text { CTGAAATTTATCACCCGCCATAGTAGACGTATCA }\end{array}$ \\
\hline CP1-S1-out & $\begin{array}{l}\text { (FAM)GCCTTCAATGAAGTGGGTCGAAAGGAGACAA } \\
\text { GGC(Dabcyl)CAGTTGAGACGAACATTCCTAAGTCTGAA } \\
\text { ATTTATCACCCGCCATAGTAGACGTATCACCAGG }\end{array}$ \\
\hline CP2-S1-in & $\begin{array}{l}\text { (FAM)AGCCTTCAATGAAGTGGGTCGAAAGGAGACA } \\
\text { AGGCT(Dabcyl)CCAGGCAGTTGAGACGAACATTCCTAA } \\
\text { GTCTGAAATTTATCACCCGCCATAGTAGACGTATCA }\end{array}$ \\
\hline CP2-S1-out & $\begin{array}{l}\text { (FAM)AGCCTTCAATGAAGTGGGTCGAAAGGAGACA } \\
\text { AGGCT(Dabcyl)CAGTTGAGACGAACATTCCTAAGTCTG } \\
\text { AAATTTATCACCCGCCATAGTAGACGTATCACCAGG }\end{array}$ \\
\hline
\end{tabular}




\begin{tabular}{|c|l|}
\hline $\begin{array}{c}\text { CP-S1-in for } \\
\text { thermo-melting }\end{array}$ & $\begin{array}{l}\text { (FAM)CCTTCAATGAAGTGGGTCGAAAGGAGACAAG } \\
\text { G(BHQ1)CCAGGCAGTTGAGACGAACATTCCTAATCT } \\
\text { GAAATTATCACCCGCCATAGTAGACGTATCA }\end{array}$ \\
\hline $\begin{array}{c}\text { CP-S1-out for } \\
\text { thermo-melting }\end{array}$ & $\begin{array}{l}\text { (FAM)CCTTCAATGAAGTGGGTCGAAAGGAGACAAG } \\
\text { G(BHQ1)CAGTTGAGACGAACATTCCTAAGTCTGAAAT } \\
\text { TTATCACCCGCCATAGTAGACGTATCACCAGG }\end{array}$ \\
\hline $\begin{array}{c}\text { CP-S1-in for } \\
\text { smFRET }\end{array}$ & $\begin{array}{l}\text { (Cy3)CCTTCAATGAAGTGGGTCGAAAGGAGACAAG } \\
\text { G(Cy5)CCAGGCAGTTGAGACGAACATTCCTAAGTCTG } \\
\text { AAATTATCACCCGCCATAGTAGACGTATCA }\end{array}$ \\
\hline smFRET & $\begin{array}{l}\text { (Cy3)CCTTCAATGAAGTGGGTCGAAAGGAGACAAG } \\
\text { G(Cy5)CAGTTGAGACGAACATTCCTAAGTCTGAAATTT } \\
\text { ATCACCCGCCATAGTAGACGTATCACCAGG }\end{array}$ \\
\hline G4 & GGGTAGGGCGGGTTGGG \\
\hline GGGTAGGGCGGGTTGGGCCAGGCAGTTGAGACGAA \\
CATTCCTAAGTCTGAAATTTATCACCCGCCATAGTAGA \\
CGTATCA
\end{tabular}




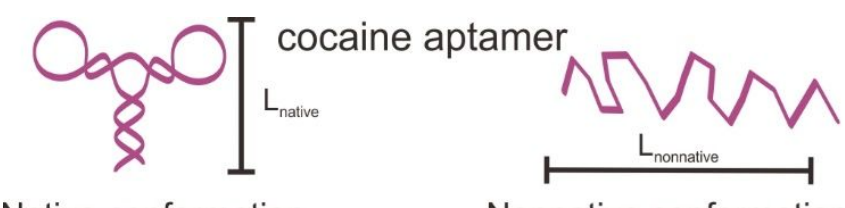

Native conformation

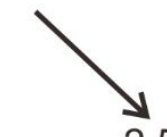

Nonnative conformation

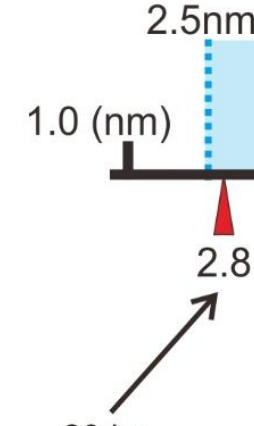

20 bp edge

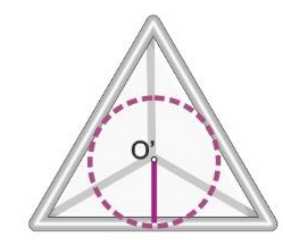

DNA Tetrahedon (This work)

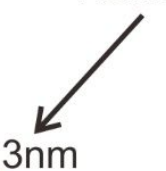

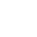




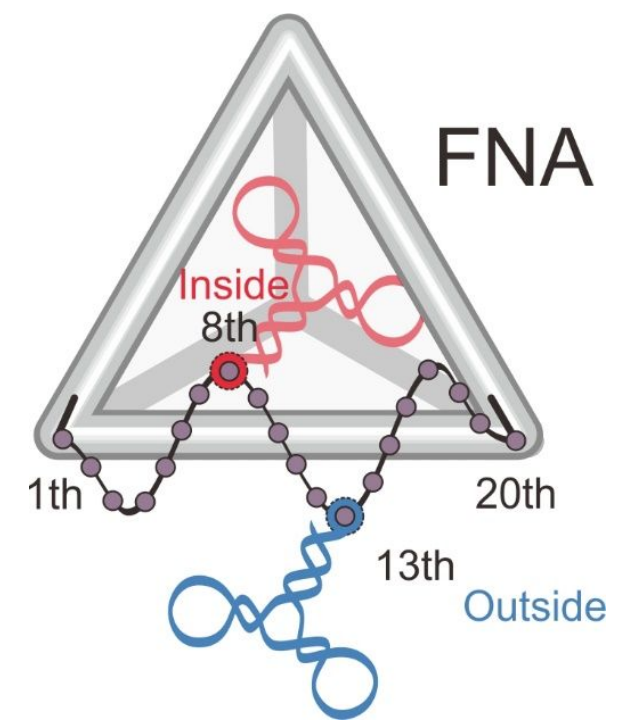

Figure S2. Schematic of the aptamer-FNA for inside and outside configruations. The position of aptamer related to the FNA is engineered by adjusting the incorporation site of aptamer along S1 DNA in the FNA. 
a) Aptamer-FNA (inside configuration)
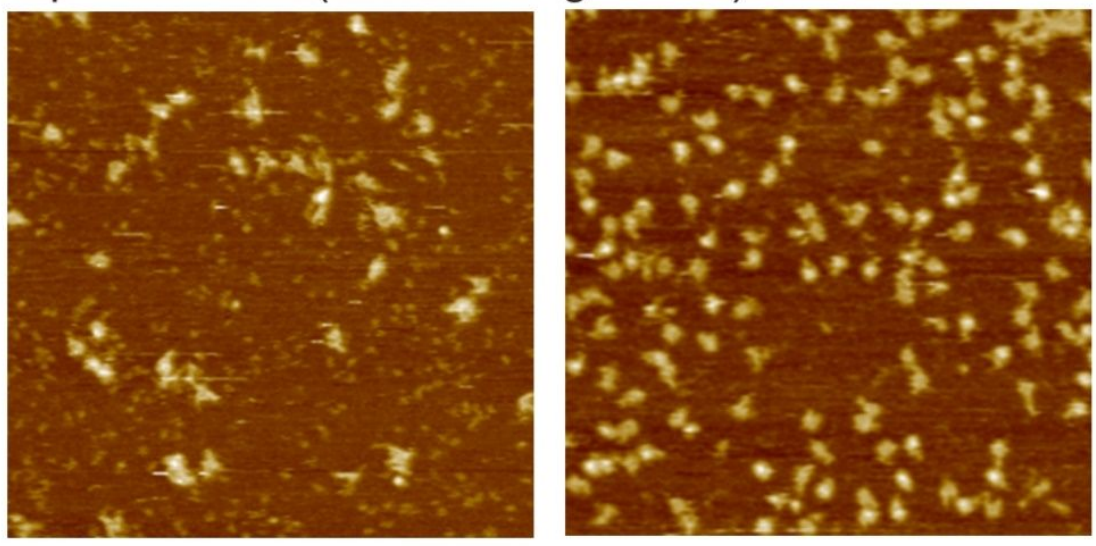

b) Aptamer-FNA (outside configuration)
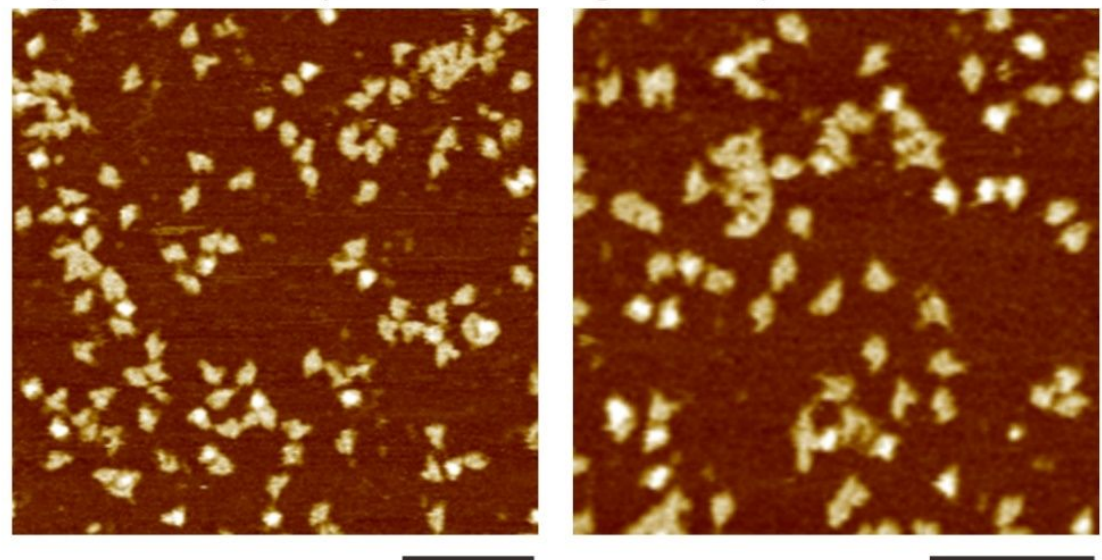

c)

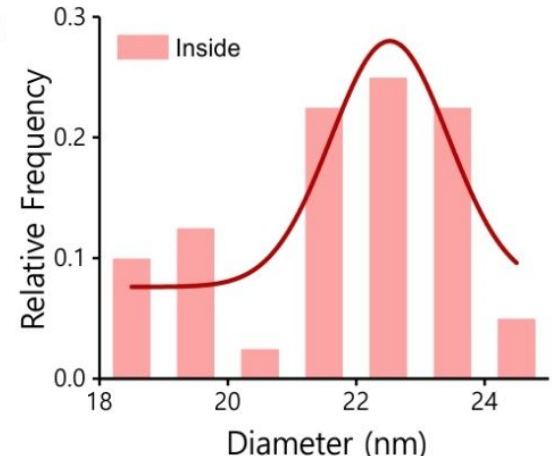

d)

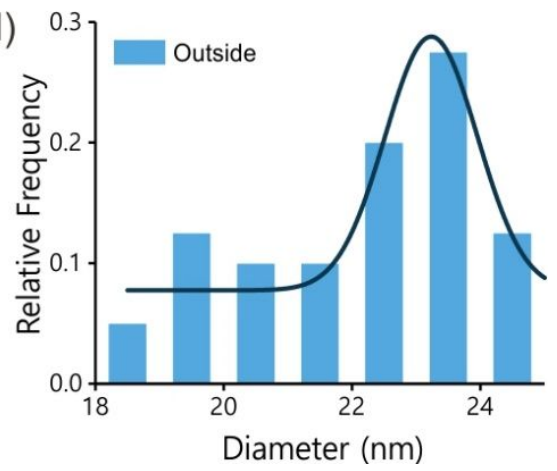

Figure S3. a-b) AFM images for aptamer-FNA with inside and outside configurations. Scale bar: 100nm. c-d) Histograms showing the size distribution of aptamer-FNAs with inside and outside configurations. Data were collected based on 50 counts of sample for each group from AFM images. 


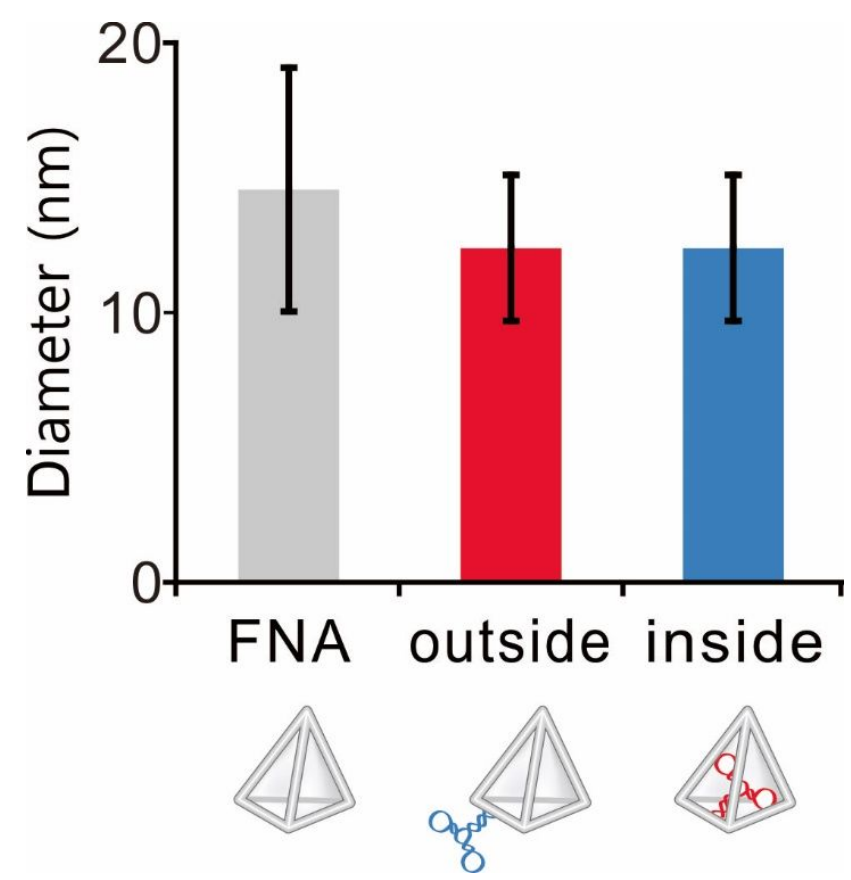

Figure S4. Histograms showing the size distribution of FNA and aptamer-FNA complex with inside and outside configurations. Data were collected based on 30 counts of sample for each group from DLS. 

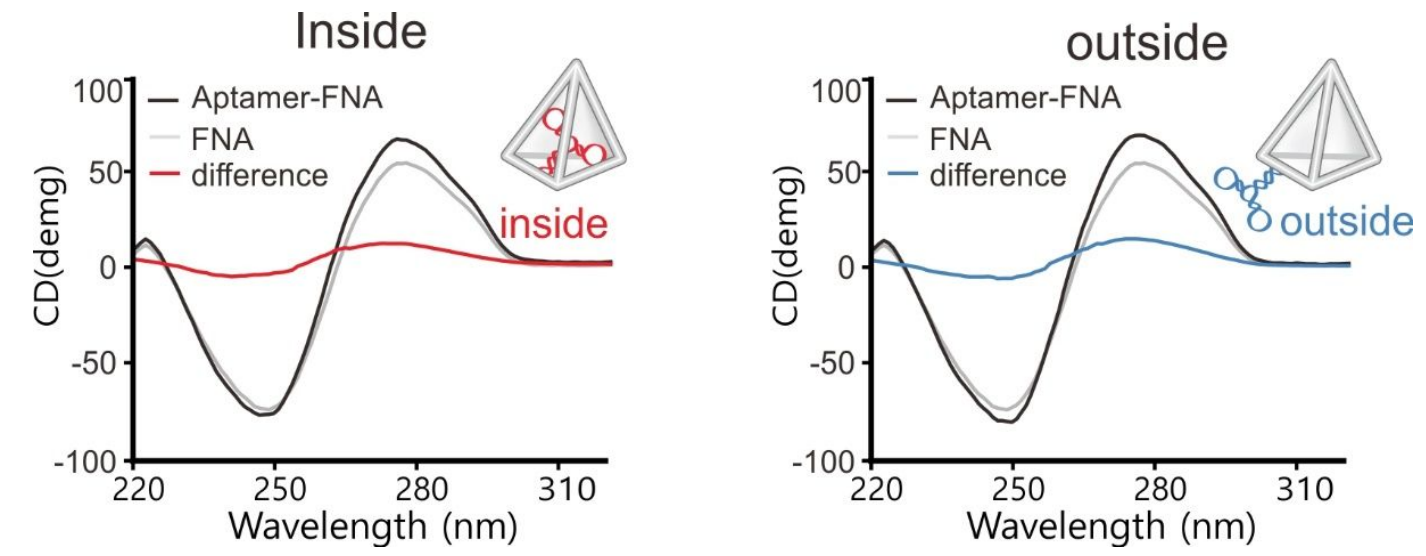

Figure S5. Characterization of incorporated aptamer by CD spectroscopy. CD spectrum of FNA, aptamer-FNA complex for inside and outside configurations, and the difference. 

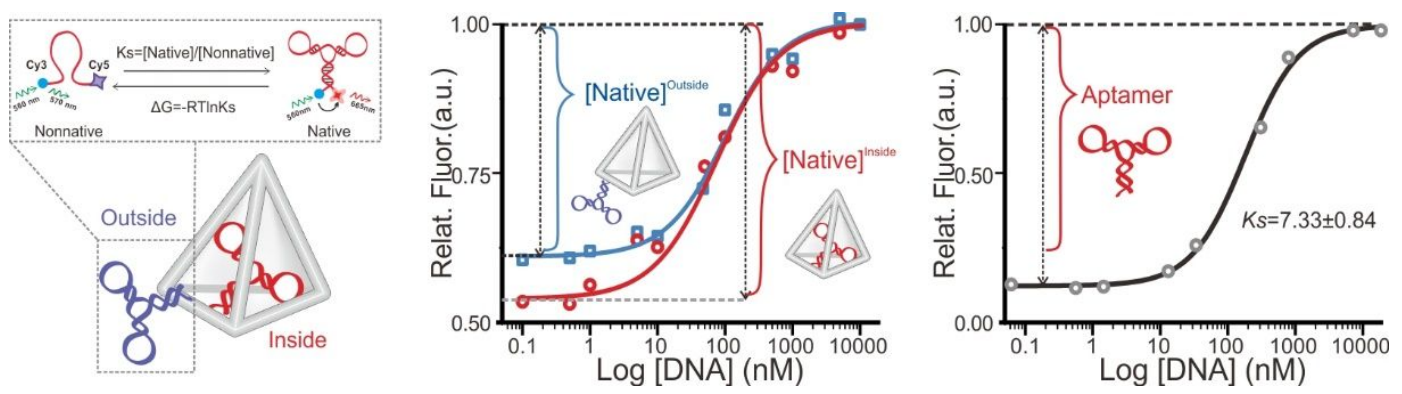

Figure S6. Schematic of showing the Ks determination of aptamer inside and outside FNA, respectively. Following the equation $\mathrm{S} 1$, the $K s$ for aptamer inside and outside FNA and plain aptamer are $0.86 \pm 0.05,0.55 \pm 0.04$ and $7.33 \pm 0.84$, respectively. 
a)

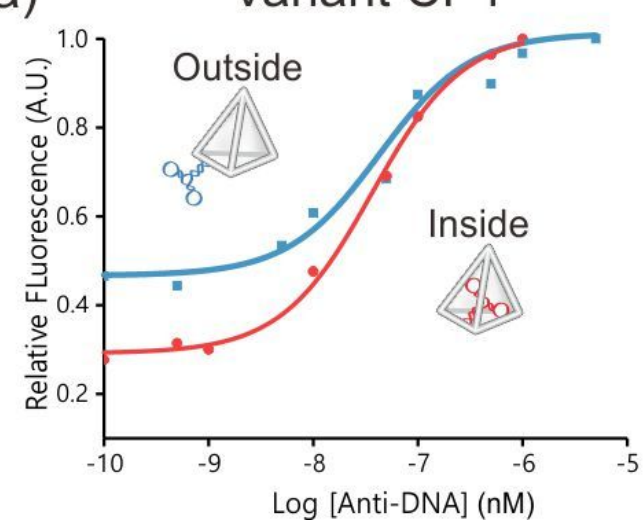

b)

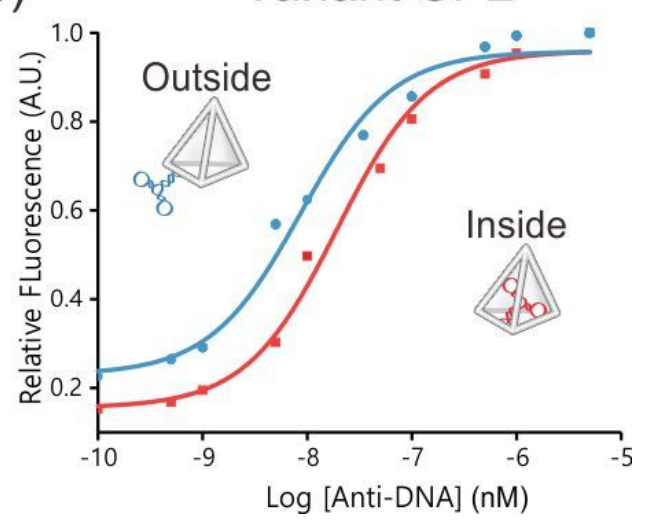

Figure S7. Schematic of showing the Ks determination of variant CP1 and CP2 inside and outside FNA, respectively. Following the equation S1, the $K s$ for aptamer inside and outside FNA are $3.26 \pm 0.32$ and $1.46 \pm 0.32$ for variant $C P 1$, and $5.19 \pm 0.16$ and 3.17 \pm 0.18 for variant $\mathrm{CP} 2$, respectively. 
a)

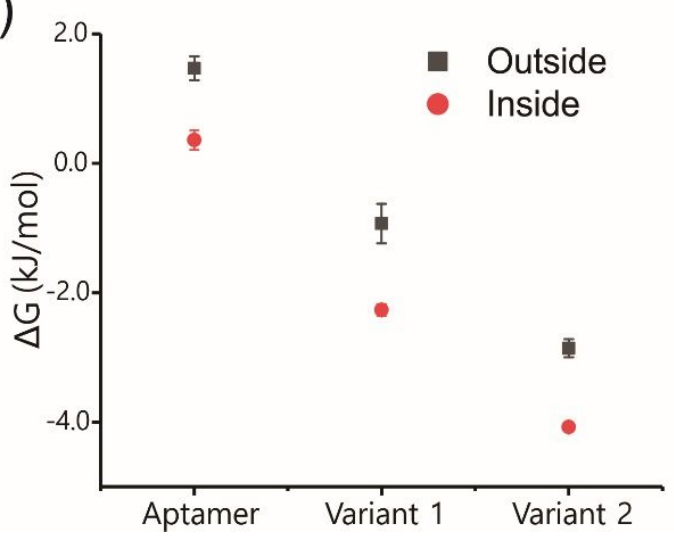

b)

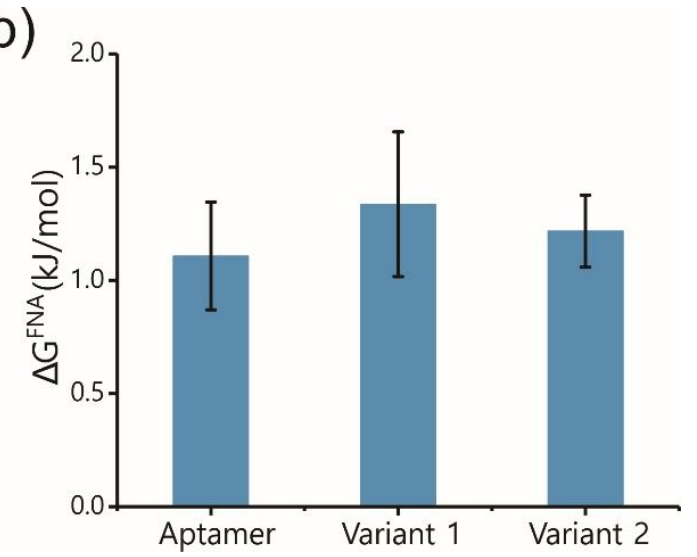

Figure S8. a, the folding free energy $(\Delta G)$ for aptamer, variant CP1 and variant CP2 inside and outside FNA. $b$, the folding free energy contribution from FNA confinement for aptamer, variant 1 and variant 2 . 


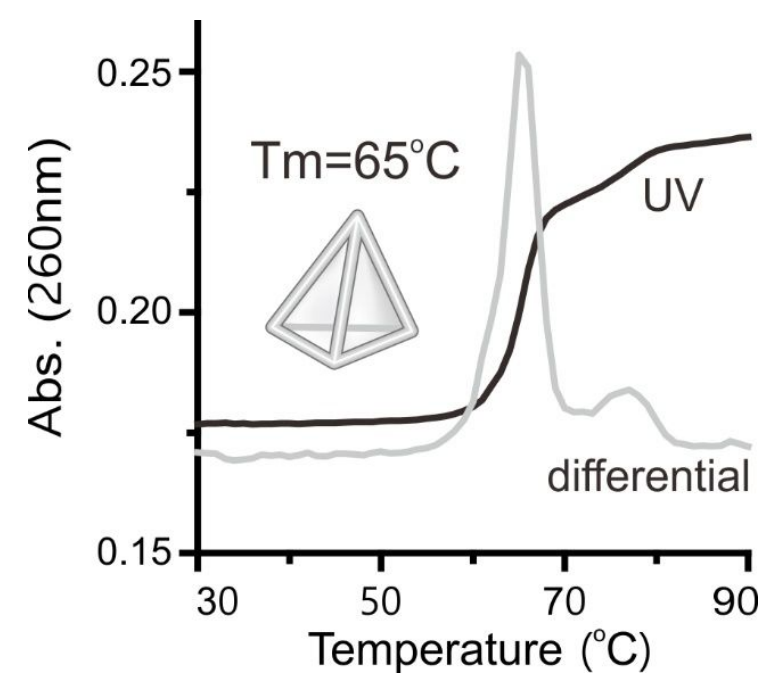

Figure S9. Thermodynamic stability analysis of FNA with thermo-melting experiments. This thermo-melting result indicated that the FNA maintain tetrahedral DNA nanostructure until 62 degree. 

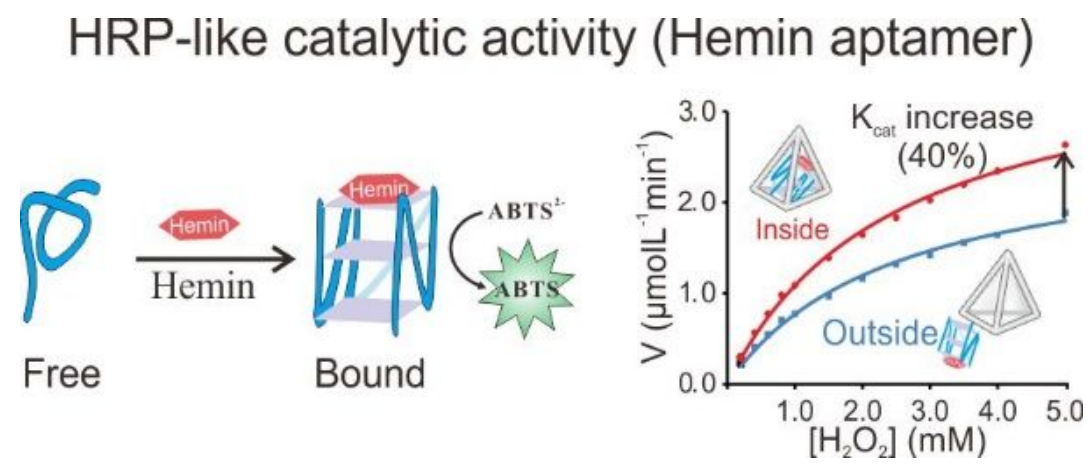

Figure S10. Catalytic activity determination of hemin aptamer incorporated inside and outside FNA. 


\section{Reference}

1. $\quad$ Lin, M.; Wang, J.; Zhou, G.; Wang, J.; Wu, N.; Lu, J.; Gao, J.; Chen, X.; Shi, J.; Zuo, X.; Fan, C. Programmable Engineering of a Biosensing Interface with Tetrahedral DNA Nanostructures for Ultrasensitive DNA Detection. Angew. Chem., Int. Edit 2015, 54, 2151-2155.

2. Chidchob, P.; Offenbartl-Stiegert, D.; McCarthy, D.; Luo, X.; Li, J.; Howorka, S.; Sleiman, H. F. Spatial Presentation of Cholesterol Units on a DNA Cube as a Determinant of Membrane Protein-Mimicking Functions. J. Am. Chem. Soc. 2019, 141, 1100-1108.

3. Jonchhe, S.; Pandey, S.; Emura, T.; Hidaka, K.; Hossain, M. A.; Shrestha, P.; Sugiyama, H.; Endo, M.; Mao, H. B. Decreased Water Activity in Nanoconfinement Contributes to the Folding of G-Quadruplex and I-Motif Structures. Proc. Natl. Acad. Sci. U. S. A. 2018, 115, 9539-9544.

4. Fu, Y.; Zeng, D.; Chao, J.; Jin, Y.; Zhang, Z.; Liu, H.; Li, D.; Ma, H.; Huang, Q.; Gothelf, K. V.; Fan, C. Single-Step Rapid Assembly of DNA Origami Nanostructures for Addressable Nanoscale Bioreactors. J. Am. Chem. Soc. 2013, 135, 696-702. 